\title{
Demand Response Process in Context of the unified LINK-based Architecture
}

\author{
A. ILO \\ TU Wien, Vienna, Austria
}

\begin{abstract}
This paper presents for the first time a unified LINK-based architecture for power systems, which provides the harmonization of power system physics and market rules. It is based on the holistic technical model of power systems "Energy supply chain net" and on the corresponding holistic power market model. The last one is illustrated by means of the actual Austrian electricity market. Furthermore, we show how the unification of the market and grid in the same flow diagram increases the uptake of demand response also in the residential, commercial and small business sectors. Their price driven demand response is described in detail.
\end{abstract}

Keywords: LINK-Paradigm, Unified LINK-based architecture, demand response, electricity market, Smart Grid.

\section{Introduction}

Demand Side Management (DSM) and Demand Response (DR) are processes which try to modify the electricity consumption shape of customers. DSM was coined following the 1970s energy crisis and since than it is continuously used by electricity utilities as an instrument on increasing efficiency and shaving peaks [1]. It includes almost medium to long term countermeasures. With the technology progress and the rise of distributed generation are opened other perspectives on demand shifting and the reduction of total energy consumption. DR rose and have been dedicated to shortterm load reduction in response to a signal from the power grid operator, or a price signal from electricity market.

Indeed nowadays, there is a very slow uptake of DR, particularly in the residential, commercial and small business sectors [2]. The proposed structures are quite complicated and requires big data exchange [3], [4], which causes the increase in the complexity of system operation etc..

This paper recommends for the first time a new simple structure, named unified LINKbased architecture, which under others enables DR process in the residential, commercial and small business sectors. The new operational architecture is based on the LINK-Paradigm and the technical and market holistic models. The last one is illustrated by means of the actual Austrian electricity market. DR is described by means of the load reduction in response to a price signal from electricity market.

\section{Holistic model}

The integration and the effective use of all available resources on the grid is possible only under a global view of power systems [5]. Figure 1 shows an overview of overall power system models. Figure 1a) shows the LINK-paradigm, which is the cornerstone 
of "Energy Supply Chain Net" overall model. LINK-paradigm is defined as a composition of an electrical appliance (be a grid part, producer or storage), the corresponding controlling schema and the LINK interface [9]. Figure 1b) shows the holistic, technical approach of power systems called "Energy Supply Chain Net" [5], which is conceived in two axis:

1. Horizontal

In the horizontal axis are set interconnected High Voltage Grids (HVG), which actually are operated from Transmission System Operators (TSO).

2. Vertical

In the vertical axis are set Medium Voltage Grid (MVG) and Low Voltage Grid (LVG), which actually are operated from Distribution System Operators (DSO) and customer plants

Power grid is arranged in autonomous parts as links in a chain net. Per definition a "Energy Supply Chain Net" is a set of automated power grids, intended for chain links, abbreviated links, which fit into one an-other to establish a flexible and reliable electrical connection. Each individual link or a link-bundle operates autonomously and have contractual arrangements with other relevant boundary links, link-bundles, and suppliers which inject directly to their own grid.

Recently power systems are perceived as assembly of four main components [7]:

1. Power plants (i.e. electricity producer);

2. Storage;

3. Grid and

4. Prosumers.

Based on LINK-paradigm, prosumers are conceived of a combination of the three first main power system components and are characterised by a new definition as follows.

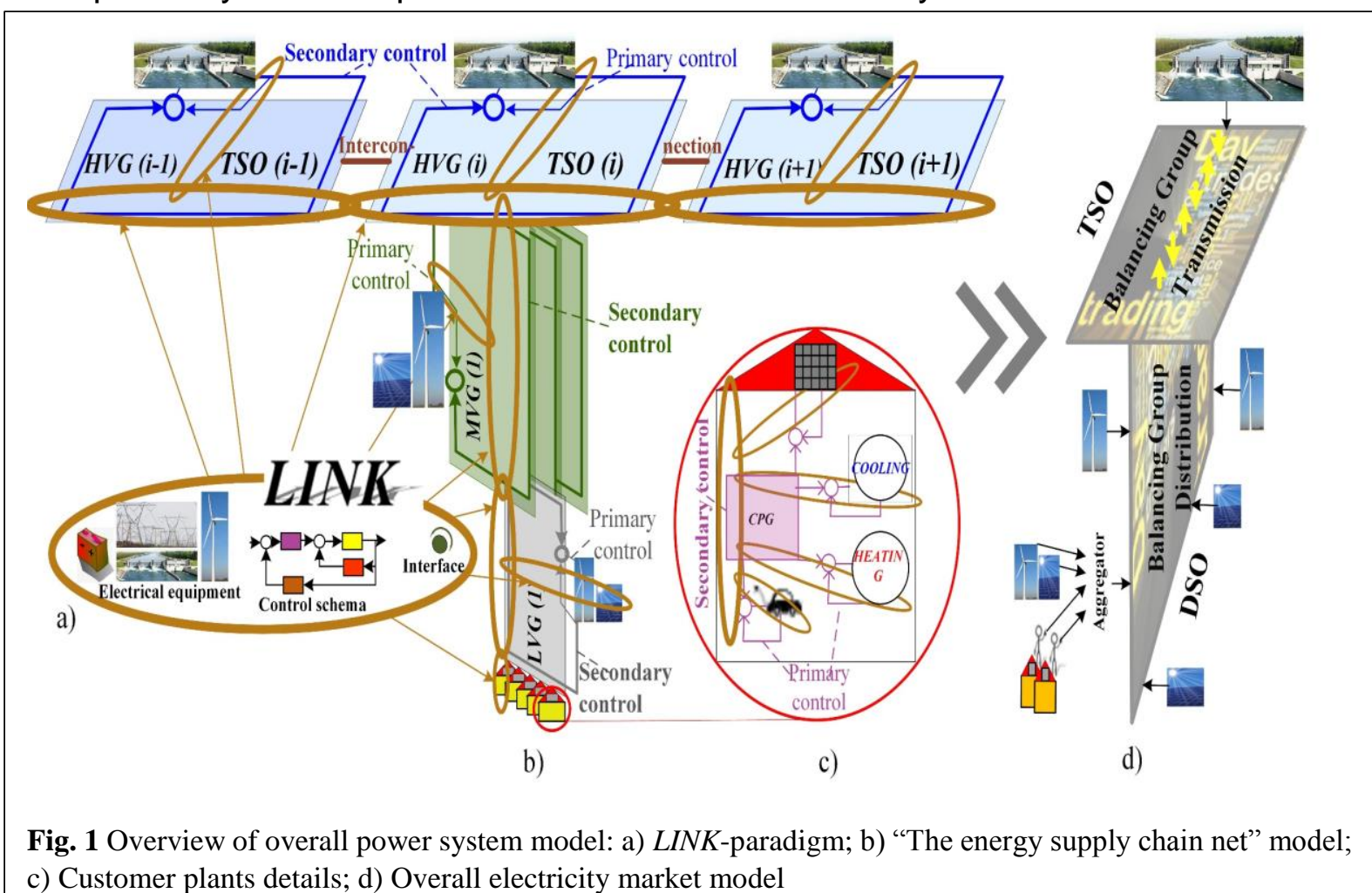

c) Customer plants details; d) Overall electricity market model 


\section{Definition}

Prosumer is a natural or legal person being owner of small electricity or/and storage facilities which are connected with each other through its own grid. He is connected to the power grid, but the produced electricity is mainly used to supply his own load. $\mathrm{He}$ is selling his electric energy surplus, and buying electric energy for own use.

Consumers are treated as a special case of prosumers and defined as follows.

\section{Definition}

Consumer is a natural or legal person buying electric energy for own use. He is connected to the power grid through its own grid.

Figure 1c) shows details of a typical residential consumer. The supplier -i.e. photovoltaic installed on the roof -, storages i.e. - electrical car battery and cooling- and heating systems - are connected with each other via house intern grid - i.e. Customer Plant Grid (CPG).

Therefore there are only three independent main power system components, which create the base for the definition of the LINK-based architecture components [6]. This three main architecture components are:

1. Producer-Link;

2. Storage-Link; and

3. Grid-Link.

As in [6] Producer-Link is defined as a composition of an electricity production facility be a generator, photovoltaic, etc., its Primary-Control and the Producer_Interface. Storage-Link is defined as a composition of a storage facility be the generator of a pump power plant, batteries, etc., its Primary-Control and the Storage_Interface. While, the Grid-Link is defined as a composition of a grid part, called Link-Grid, the corresponding Secondary-Control and Grid_Interface. Each architecture component has also its own operator - i.e. the Producer-Link has the Electricity Producer Operator; the Storage-Link has the Storage Operator (StO) while the Grid-Link has the Grid-LinkSystem_Operator.

Figure 1d) shows an overview of the overall electricity model, which is a mirror of the technical overall model. Based on this model not only the TSO [6], [8], who operates on the horizontal axis of power systems, but also the DSO, who operates on the vertical axis of power systems, will communicate directly with the market and take over the task of load-power injection balance. And in fact two of the main tasks of Grid-LinkSystem_Operator (Grid-Link-SO) defined in [6] are: Facilitation of an effective and wellfunctioning retail market and load-power injection balancing in real time. The owner of the decentralised appliances may participate in to the market directly or through an aggregator.

\section{Unified LINK-based architecture}

LINK-paradigm applies particularly to network operation. When the structure of electricity supply changes so much, due to many decentralised generation units, each with the possibility to interfere with the system operation at all voltage levels, then it is needed a new architecture in order to utilize this flexibility, so that the power system operation can remain reliable. The 


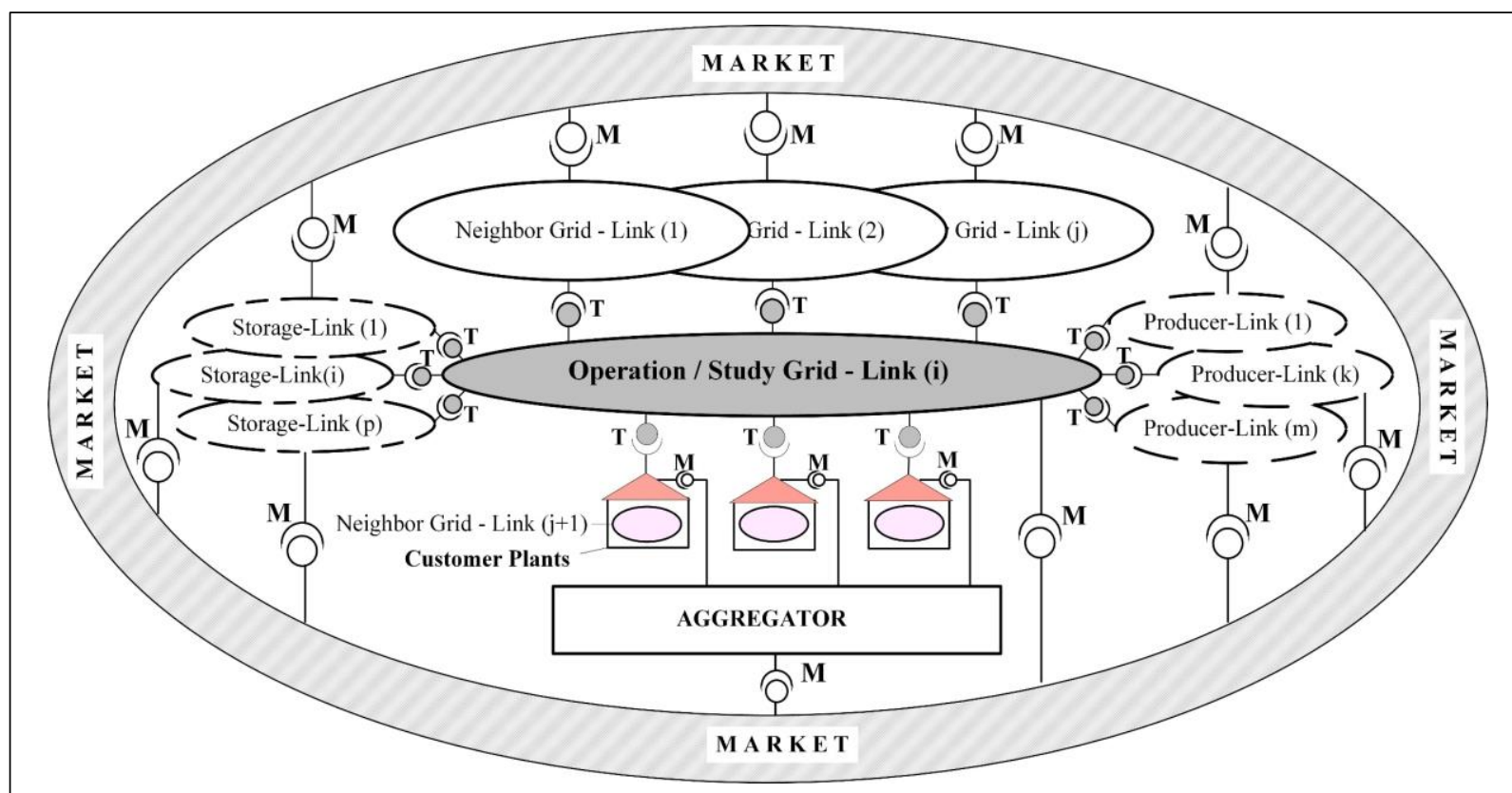

Fig. 2 Overview of the unified $L I N K$-based architecture of smart power systems

new operational architecture of power systems should guarantee that it performs as expected by unifying the operation processes and systematising the execution of operational tasks.

\section{Definition}

The unified LINK-based architecture is an architecture of power systems in which all relevant components like electricity:

- Producer (independent from the used technology or size, ex. big power plants, decentralised generations, etc.)

- Storage (independent from the used technology or size, ex. pumped power plants, batteries, gas systems etc.)

- Grid (including all voltage levels - i.e. high-, medium- and low voltage grid -)

- Market

are merged into one single structure. This architecture unifies all interactions within the power system itself and between it and market thus creating the possibility to harmonize them.

Figure 2 shows an overview of the unified LINK-based architecture of smart power systems. Operation or study Grid-Link communicates via technical interfaces $T$ with other neighbour Grid-Links, and Producer- and Storage-Links which are connected to its own grid. Technical interfaces are already well defined [6]. Their communication with the electricity market is foreseen through the market interface $M$. The illustration of market interface $M$ is done based on the Austrian electricity market [8]. Some of the most prominent participants in this market are:

- Control Area Manager, CAM $\rightarrow$ responsible for load-frequency control within its control area

- System Operator, SO $\rightarrow$ operator of transmission or distribution grid

- Supplier $\rightarrow$ commercial provider of electric energy

- Consumer $\rightarrow$ buyer of electric energy for own use

- Clearing and Settlement Agent, CSA (balance group coordinator) $\rightarrow$ responsible of organising, clearing and settling of balance within a control area 
- Balance responsible Party, BRP balance group representative $\rightarrow$ an entity representing a balance group vis-à-vis other market participants and vis-à-vis the CSA

In Austrian electricity market were introduced balance groups to enable consumers, generators, suppliers and wholesalers to trade or conclude deals with each other [8]. Whoever takes electricity off the power grid, feeds in or trades must be a member of a balance group. BRP balances the load within the balance group. CSA takes care for load balancing within the control area, which includes more than one balancing group, up to the intraday trade. In the following CAM ensures the system stability in real time by performing the load-frequency process, which in reality is handled by TSOs. Consequently, TSO assumes a new role, the one of CAM. The number of the data that should be sent to CAM or rather to TSO is immense [7], [8]. Beside the different contracts types -i.e. utilisation-, supply- or storage contract -, meter readings and bills, market participants exchange schedules for electricity trading [8]. Here it should be noted that there are two types of schedules:

1. Internal

Internal schedules for electricity trading between balance groups in the sane control area

\section{External}

External schedules for electricity trading between balance groups in different control areas

Figure 3 shows a schematic presentation of the Austrian electricity market model in contest of the unified LINK-based architecture. In this figure are shown the contractual relations and information exchange among market participants. Market participants and their roles remain

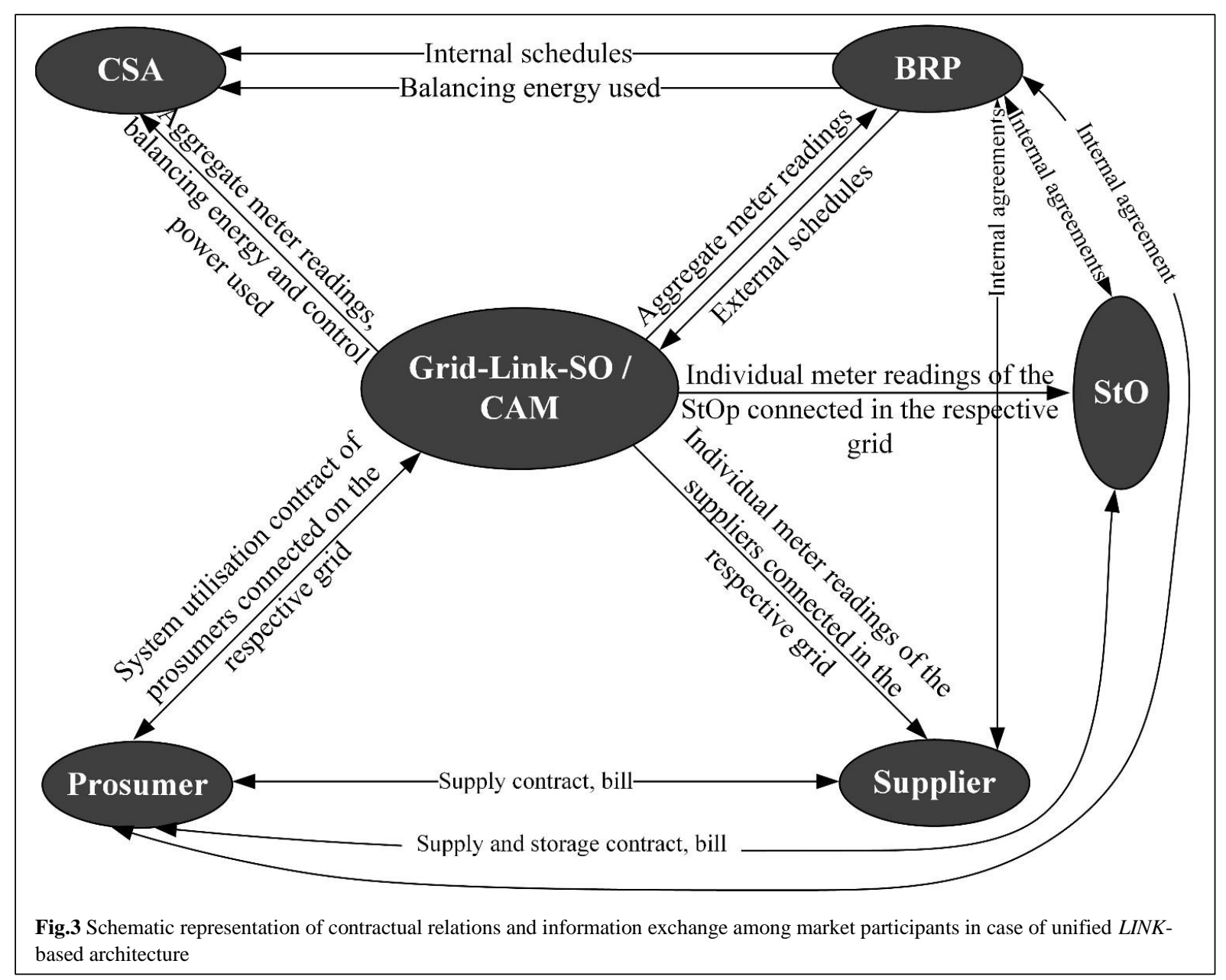


almost the same as in [8] up to the roles of TSO and DSO combined with CAM. With the unified LINK-based architecture are introduced three crucial novelties in the electricity market model:

1. Storage Operator

Storage operator rice is a logical consequence of the new technological developments and is defined as follows:

\section{Definition}

Storage Operator, StO is a natural or legal person that provides and stores electric energy to or from other natural or legal persons.

StO must participate to a balance group, have supply and storage contracts with the prosumers, and receives the individual meter readings from the grid, where it is connected.

2. Consumers are transformed to prosumers

In recent years, many consumers have gradually been transformed into prosumers. Their inclusion on the market model is essential for the fair development of the electricity market.

3. Adoption of the CAM role

The adoption of the CAM role is crucial for a secure, reliable and efficient operation of power systems by fulfilling the stringent requirements of data privacy and cyber security. In the new market model each of the Grid-Link-SO - i.e. TSO and DSO assumes the new role CAM. That means that each Grid-Link-SO have also the CAM role, and is responsible for the load balance process in their own control area up to the real-time time frame. The technical realization is realistic, because a secondary control for the active power and frequency is designed per each Grid-Link type [4], [6]

In this new electricity market model the scheduling process is foreseen to remain the same as described in [8] with the only difference that the pumping schedules should be converted to storage schedules.

\section{Price driven demand response}

The unified LINK-based architecture allows the properly launch of demand response. The emergency DR - i.e. load reduction to alleviate overloading in high voltage grid, which is triggered from the power grid operator - is described in [6]. Heir we will focus on DR to a price signal from electricity market.

The activation of the residential, commercial and small business sectors, which join the real-time pricing demand response through already concluded contracts, may be triggered at any time. Their degree of participation in the demand response process may be different depending on the time of the day, duration interval, price value, etc. Let's assume that conditioned from weather and the minimal load consumption there do exist a surplus of the electricity in the market, which causes a price decrease. Figure 4 shows the information flow during price driven demand response. The new, reduced price is sent via the aggregator through the market interface $M$ to the House Management Units (HMU), [6], of each customer. Figure 5 shows a detailed view in customer- and low voltage level of the information flow by a price reduction signal. $\mathrm{HMU}$ after having calculated the possibilities of demand increase in the house, sends 


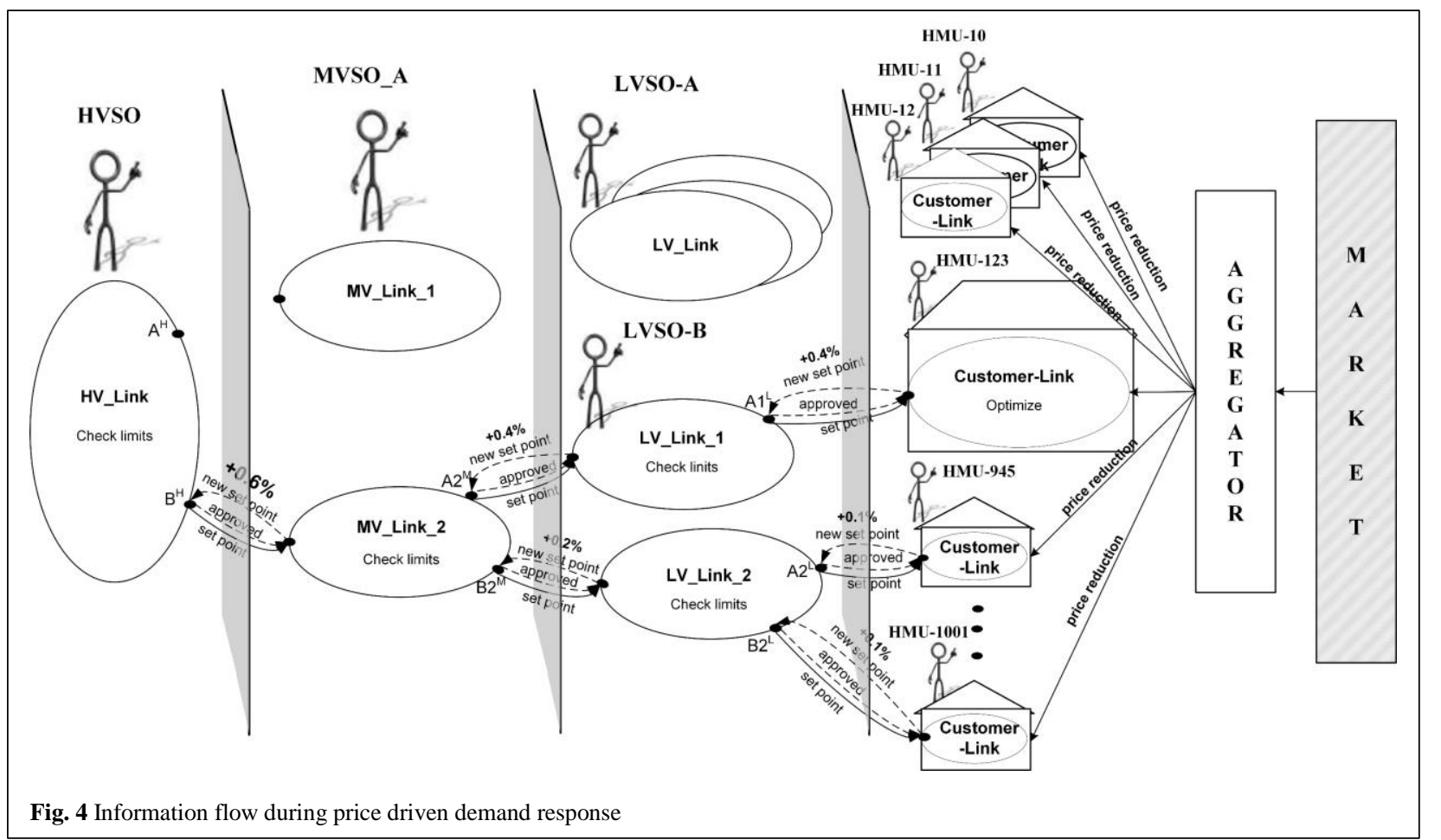

a request to increase the consumption by $0.4 \%$ via the technical interface $T$ to the boundary node $A 1 L$ of LV_Link_1. After receiving the request for the new set point, Low Voltage Grid-Link System Operator B (LVSO-B) check power flow limits in the own Grid-Link. In the case that the power exchange in the boundary node $\mathrm{A}^{\mathrm{M}}{ }^{\mathrm{M}}$ with the Medium Voltage_Link_2 is affected, he should pass over the request on to the Medium Voltage System Link-Grid Operator-A (MVSO-A). After have collected all incoming requests MVSO-A calculates power flow in the own Grid-Link. Based on calculations results he sends on to the High Voltage Grid-Link System Operator (HVSO) a flow increase request of $0.6 \%$ in the boundary node $\mathrm{B}^{\mathrm{H}}$. After have collected all incoming

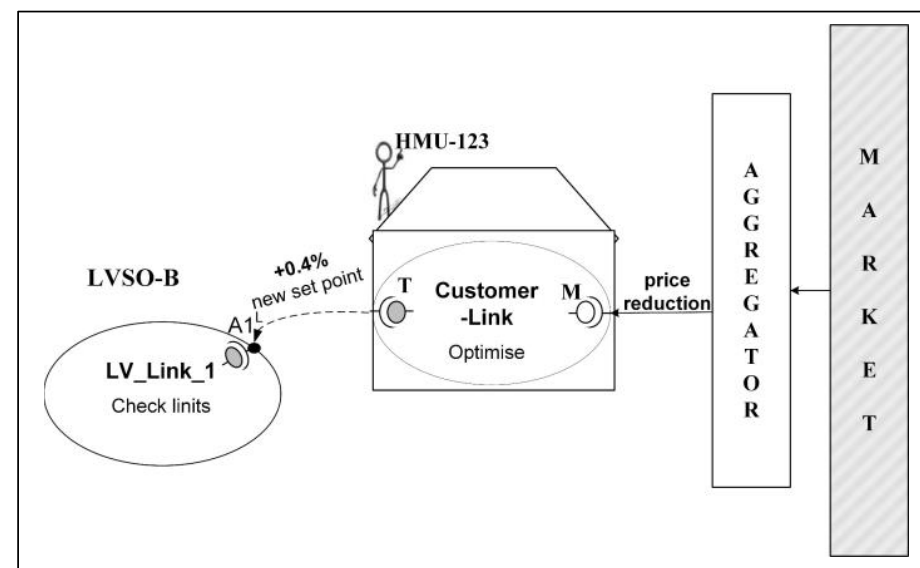

Fig. 5 Detailed view of the information flow by a price reduction signal (customer and low voltage level) requests HVSO performs all necessary calculations - i.e. power flow, n-1 security, etc. -. After having checked all limits HVSO approves the new set points and notify MVSO-A. The last one approves the new set points in boundary nodes $\mathrm{A} 2^{\mathrm{M}}$ and $\mathrm{B}{ }^{\mathrm{M}}$ and notifies LVSO-B. The last one approves the new set points in boundary nodes $A 1^{L}$, and $A 2^{L}$ and $\mathrm{B} 2^{\mathrm{L}}$ and notifies the respective HMUs, which execute the demand increase.

The one flow diagram of demand response in emergency- and price driven cases enable residential, commercial and small business sectors to perceive transparent energy prices and to contribute in the reliable and efficient operation of electric power system. 


\section{Conclusions}

The unified LINK-based architecture merges producers, storages, grid and market into one single structure. Prosumers and Storage appliances are newly, more precisely defined. The market $L I N K$-based model is extended with new actors and their old roles are redistributed. The demand response for residential, commercial and small business sectors is described in a unified flow diagram. Demand response can be triggered from the grid operator in emergency cases or market through different price incentives.

\section{References}

[1] Gellings C.W. and Parmenter K.E. (2016) Demand-Side Management. In: Energy Efficiency and Renewable Energy Handbook, Second Edition

[2] Strbac G. (2008) "Demand side management: Benefits and challenges". In: Energy Policy, Volume 36, Issue 12, December, Pages 4419-4426.

[3] Raab A. F., Ferdowsi M., Karfopoulos E., Unda I., Skarvelis-Kazakos S., Papadopoulos P., Abbasi E., Cipcigan L., Jenkins N., Hatziargyriou N., and Strunz K. (2016) Virtual power plant control concepts with electric vehicles. In Intelligent System Application to Power Systems (ISAP), 2011 16th International Conference September, pp. 1-6.

[4] Etherden N., Vyatkin V., Bollen H.J. (2015) Virtual Power Plant for Grid Services using IEC 61850. In: IEEE Transaction on Industrial Informatics, DOI 10.1109/TII.2414354, p. 1-11.

[5] Ilo A. (2013) The Energy Supply Chain Net. In: Energy and Power Engineering - Journal - Scientific Research Publishing, [Online]. Volume 5, July, pp. 384390.

[6] Ilo A. (2016) "Link" - the Smart Grid Paradigm for a Secure Decentralized Operation Architecture. In Electric Power Systems Research - Journal Elsevier, Volume 131, pp. 116-125.

http://www.scirp.org/journal/PaperInformation.aspx?PaperID=34182

[7] Ilo A. (2016) Minimization of exchanged data on the TSO-DSO cross border by application of a new operation architecture. In: CIGRE International Colloquium, 2-3 November, Philadelphia, USA.

[8] E-control (2013) The Austrian electricity market. In: https://www.econtrol.at/documents/20903/-/-/f007c31e-b6b9-48a1-83a1-53212aae5365 